\title{
Extracción broncoscópica de cuerpos extraños en la vía aérea. Diez años de experiencia
}

\author{
Dr. José David Palmer-Becerra, ${ }^{*}$ Dra. Carmen María Vania Osorio-Rosales, ${ }^{* *}$ Dr. Jorge Eduardo Madriñán- \\ Rivas, ${ }^{* * *}$ Dr. Arturo Montalvo-Marín, ${ }^{* * * *}$ Dr. Hugo Uro-Huerta*****
}

\section{RESUMEN}

Introduccion. La aspiración de un cuerpo extraño es un evento común en niños y es una causa frecuente de morbilidad y mortalidad, la que se debe a la dificultad respiratoria; ésta puede ser súbita, acompañada de tos.

Objetivo: Determinar las características clínicas y los resultados del tratamiento broncoscópico en niños que han aspirado cuerpos extraños.

Material y metodo. Estudio retrospectivo de enero del 2000 a diciembre del 2009 de 84 pacientes que aspiraron un cuerpo extraño, a quienes se le extrajeron por vía broncoscópica. Fueron 55 niños y 29 niñas cuyas edades iban de ocho meses a 13 años.

Resultados: Las manifestaciones clínicas más frecuentes fueron la asfixia en 75 (90\%), la cianosis, 67 (80.4\%) y la polipnea en 63 (75.6\%). Los datos radiológicos fueron positivos en 82 pacientes (98.4\%). El objeto aspirado más común fue el cacahuate (33.6\%). Los cuerpos extraños se localizaron en la tráquea en nueve casos (14.4\%); en la carina en cuatro (4.8\%); en el bronquio derecho en 52 pacientes $(62.4 \%)$ y en el bronquio izquierdo en $19(22.8 \%)$.

Conclusiones: Los cuerpos extraños en la vía aérea son una urgencia que amenaza la vida. La historia clínica es la clave del diagnóstico. La broncoscopia con anestesia general es un procedimiento seguro y eficaz.

Palabras clave: Cuerpo extraño, aspiración, broncoscopia, morbilidad, mortalidad, complicación.

\section{ABSTRACT}

Introduction. Foreign body aspiration is a common accident in children and represents an important cause of morbidity and mortality. The onset of respiratory distress may be sudden with cough.

Objective. To determine the clinical characteristics and the results of bronchoscopic treatment of children who aspired a foreign body.

Methods: Retrospective study of 84 children from January 2000 to December 2009 who had a foreign body removed from the airway by bronchoscopy. Fifty-five boys and 29 girls were included. Their ages ranged from 8 months to 13 years.

Results: The most frequent clinical manifestation was choking in 75, (90\%); cyanosis, 67 (80.4\%) and polypnea in 63 (75.6\%). Positive radiological findings were present in 82 patients (98.4\%), and the most common object aspired was a peanut (33.6\%). Foreign bodies were located in the trachea in 9 cases (14.4\%); in the carina in 4 cases $(4.8 \%)$; in the right bronchus in 52 patients $(62.4 \%)$ and in the left bronchus in 19 cases $(22.8 \%)$.

Conclusions. Airway foreign bodies are a potentially life-threatening event. Medical history is the key for the diagnosis. Broncoscopy under general anesthesia is a very safe y effective procedure for patients with airway obstruction caused by a foreign body.

Key words: Foreign bodies, aspiration, bronchoscopy, morbidity, mortality, complications.

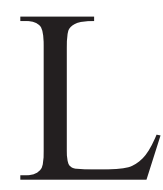

a aspiración de un cuerpo extraño que produce obstrucción de la vía aérea deteriora de manera aguda la función respiratoria y es causa de muchas muertes accidentales en el mundo. Ocurre

\footnotetext{
* Jefe de Residentes en Cirugía Pediátrica.

** Residente de 2do. Año en Cirugía Pediátrica

*** Jefe del Servicio de Endoscopia Pediátrica.

**** Profesor Titular del Curso de Cirugía Pediátrica. UNAM

***** Profesor Adjunto del Curso de Cirugía Pediátrica. UNAM
}

Correspondencia: Dr. José David Palmer-Becerra. Hospital del Niño "Dr. Rodolfo Nieto Padrón" Villahermosa Tabasco México. Calle Coronel Gregorio Méndez Magaña \# 2832, Colonia Atasta. CP 86110. Villahermosa Tabasco México. con mayor frecuencia en menores de cinco años; el mayor riesgo de que haya complicaciones se ve en menores de dos años. El problema predomina en varones. En niños mayores de seis años, el objeto aspirado de tipo inorgánico

Correo electrónico: drjosepalmer@hotmail.com Recibido: febrero, 2010. Aceptado: abril, 2010.

Este artículo debe citarse como: Palmer-Becerra JD, OsorioRosales CMV, Madriñán-Rivas JE y col. Extracción broncoscópica de cuerpos extraños en la vía aérea. Diez años de experiencia. Acta Pediatr Mex 2010;31(3):102-107.

www.nietoeditores.com.mx 
es el más común (90\%). Hasta $80 \%$ de los cuerpos extraños en vía aérea son de origen vegetal: frutas secas, cacahuate, semillas, etc. La manifestación clínica varía, desde una forma asintomática o con tos hasta cianosis de instalación súbita, polipnea y dificultad respiratoria. La broncoscopia rígida es el procedimiento diagnóstico y terapéutico de elección para la extracción. Las complicaciones pueden ir desde atelectasias, neumotórax, enfisema subcutáneo, insuficiencia respiratoria aguda, hasta los casos graves, con paro cardiorrespiratorio y muerte. El objetivo de este estudio es determinar las características clínicas y los resultados del tratamiento broncoscópico de pacientes con aspiración de cuerpos extraños en la vía aérea.

\section{MATERIAL Y METODOS}

En el Hospital del Niño "Dr. Rodolfo Nieto Padrón" de Villahermosa Tabasco, México se revisaron los expedientes de pacientes con diagnóstico de aspiración de cuerpo extraño en vía aérea en un periodo de 10 años, desde enero del 2000 a diciembre del 2009. Se hallaron 84 expedientes exclusivos de dicho hospital. Los datos que se recolectaron se estudiaron en forma retrospectiva, descriptiva y observacional. Las características clínicas tomadas en cuenta fueron edad, sexo, síntoma de presentación, examen físico, tiempo de evolución, estudio radiológico de apoyo inicial, procedimiento endoscópico realizado, localización del cuerpo extraño y complicaciones. El procedimiento se realizó con broncoscopio rígido Storz tomando en cuenta su diámetro interno coincidente con la edad del paciente. Se emplearon medidas de tendencia central.

\section{RESULTADOS}

La edad de los pacientes fue de ocho meses a 13 años con un promedio de seis años cinco meses; del sexo femenino $29(34.8 \%)$ y masculino 55 (66\%). El síntoma de presentación fue la dificultad respiratoria en 80 pacientes (96\%); datos de eventos de asfixia en 75 (90\%); cianosis en 67 (80.4\%); polipnea en 63 (75.6\%); estridor en 12 (14.4\%), atelectasia persistente en ocho (9.6\%), crisis asmática en seis $(7.2 \%)$ y asintomático con antecedentes de haber ingerido un cuerpo extraño a vía aérea en cuatro $(4.8 \%)$. El examen físico fue anormal en 80 pacientes (96\%); el signo principal fue la hipoventilación. La evaluación clínica fue normal en cuatro (4.8\%). El tiempo de evolución varió de una hora a 14 días, con promedio de siete días. Las radiografías de tórax postero-anterior y lateral fueron anormales en 82 pacientes (98.4\%); había sobredistension pulmonar en 74 (88.8\%); cuerpo extraño radiopaco en 12 (14.4\%) (Figura 1); atelectasia apical derecha en ocho (9.6\%); enfisema subcutáneo en tres (3.6); neumomediastino en tres (3.6\%). Se realizó broncoscopia en los 84 pacientes. La localización del cuerpo
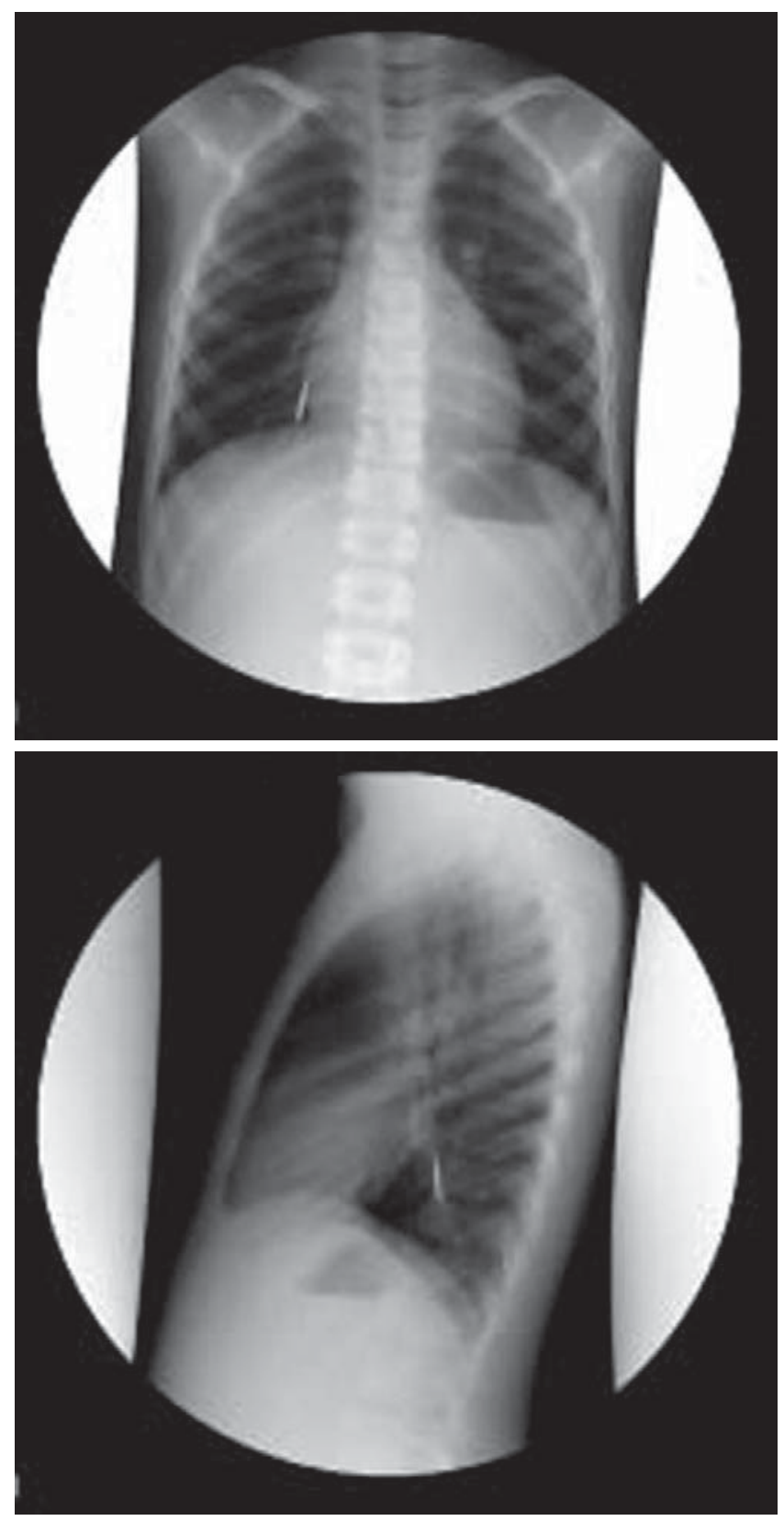

Figura 1. Radiografías postero-anterior y lateral de tórax que muestran una aguja en las parte inferior del bronquio derecho. 
extraño fue en tráquea en nueve (14.4\%); en la carina en cuatro (4.8\%); en el bronquio derecho en $52(62.4 \%) \mathrm{y}$ en el bronquio izquierdo en 19 (22.8\%). El tipo de cuerpo extraño alojado en la vía aérea fue de origen orgánico en 57 niños $(68.4 \%)$ de los cuales $28(33.6 \%)$ eran cacahuates, semillas de maíz en 11 (13.2\%) (Figuras 2 y 3), semillas de frijol en siete (8.4\%); semillas de sandia en tres (3.6\%), restos de arroz en dos $(2.4 \%)$, cáscara de pistache en dos (2.4\%) hueso de pollo en dos (2.4\%), semilla de pimienta en uno $(1.2 \%)$ y restos de carne en uno (1.2\%). Los objetos inorgánicos hallados en 27 niños (32.4\%) fueron: 14 (16.8\%) metálicos: tachuela, aguja, alfiler, clavo, arete, clip, silbato (Figuras 4, 5); tapones de plástico en ocho $(9.6 \%)$; tres $(3.6 \%)$ con restos de plástico de un globo, uno $(1.2 \%)$; botón de ropa en uno (1.2\%). Las complicaciones que ocurrieron fueron: endobronquitis en $36(43.2 \%)$; insuficiencia respiratoria que requirió ventilación mecánica en siete (8.4\%); neumonía en cinco (6\%); sangrado leve en tres $(3.6 \%)$ y absceso pulmonar apical derecho en uno (1.2\%). La evolución de los 84 pacientes fue favorable; sólo el paciente con absceso pulmonar tuvo que ser tratado con antimicrobianos por vía intravenosa durante tres semanas, inicialmente con un esquema de penicilina $\mathrm{G}$ sódica cristalina y posteriormente con cefotaxima a 150 mg por kilogramo por día y dicloxacilina a $200 \mathrm{mg}$ por kilogramo. El paciente egresó en buen estado.

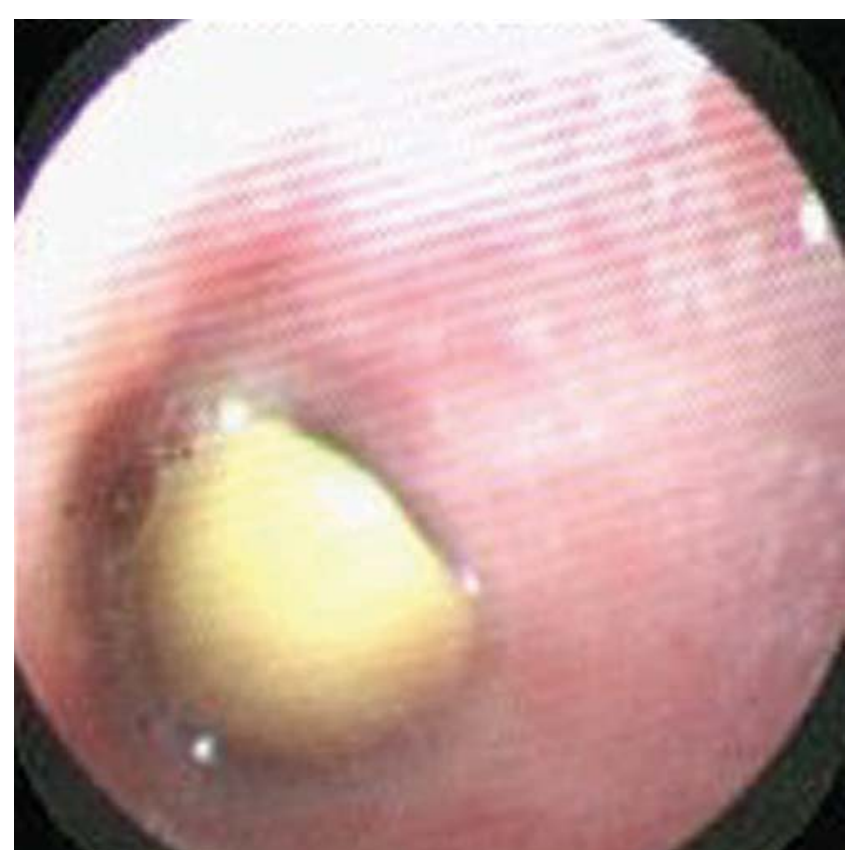

Figura 2. Se observa una semilla de cacahuate alojada en bronquio derecho.

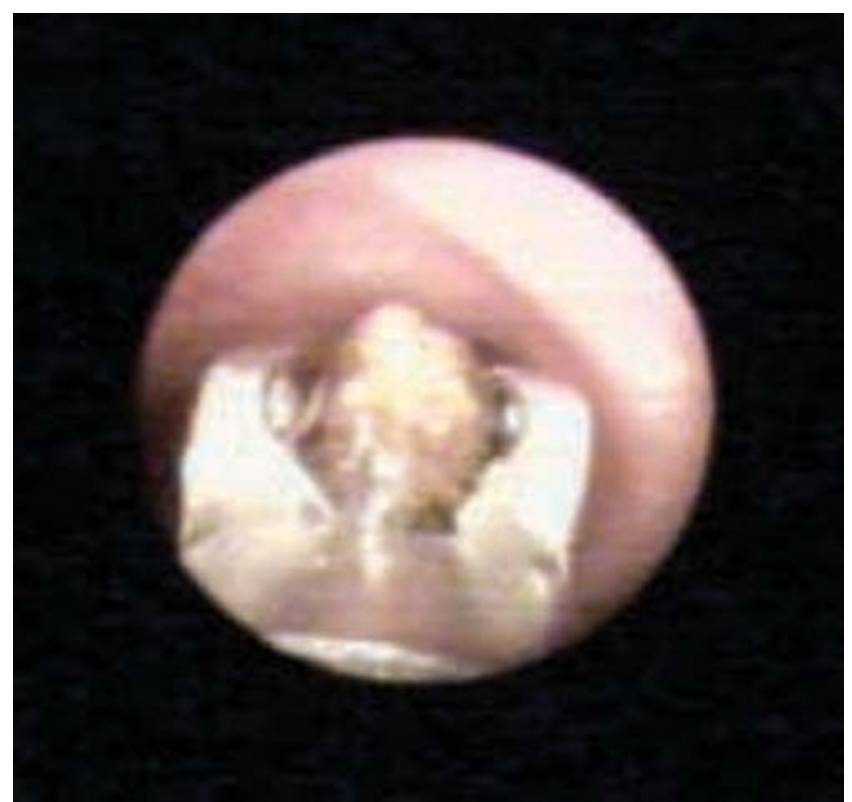

Figura 3. Momento de la extracción de un grano maíz de bronquio derecho.

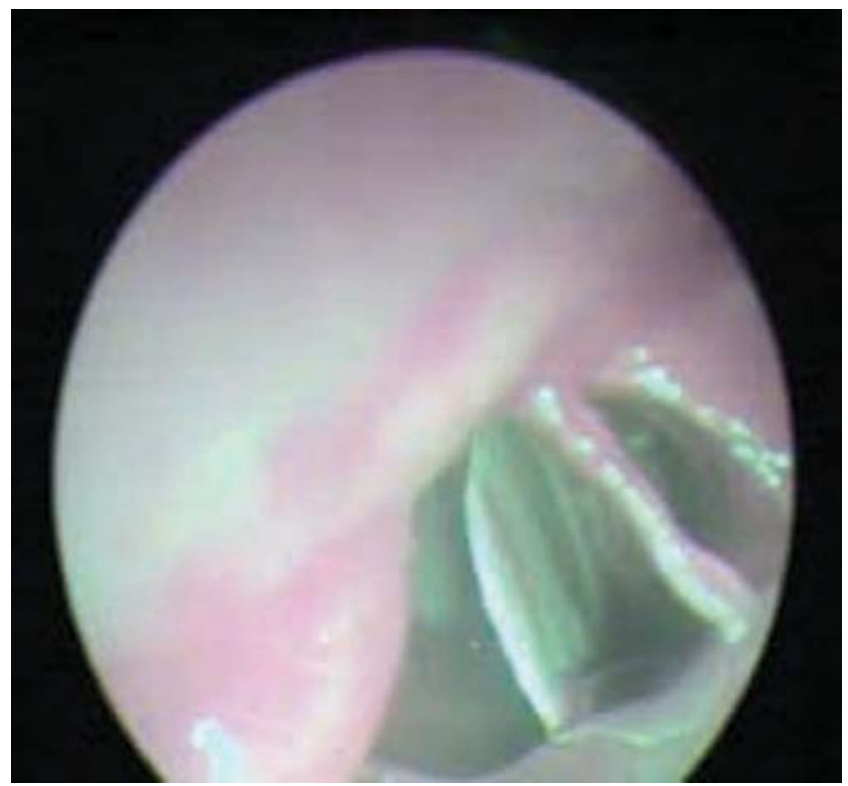

Figura 4. Se visualiza un silbato metálico en bronquio derecho.

\section{DISCUSIÓN}

Históricamente, los cuerpos extraños en la vía aérea han sido una causa muy frecuente de morbilidad y mortalidad en todo el mundo. En niños, la mayoría ocurre entre nueve y 30 meses de edad; el varón se afecta dos veces más ${ }^{1}$. El sitio más estrecho de la vía aérea es el espacio subglótico, 


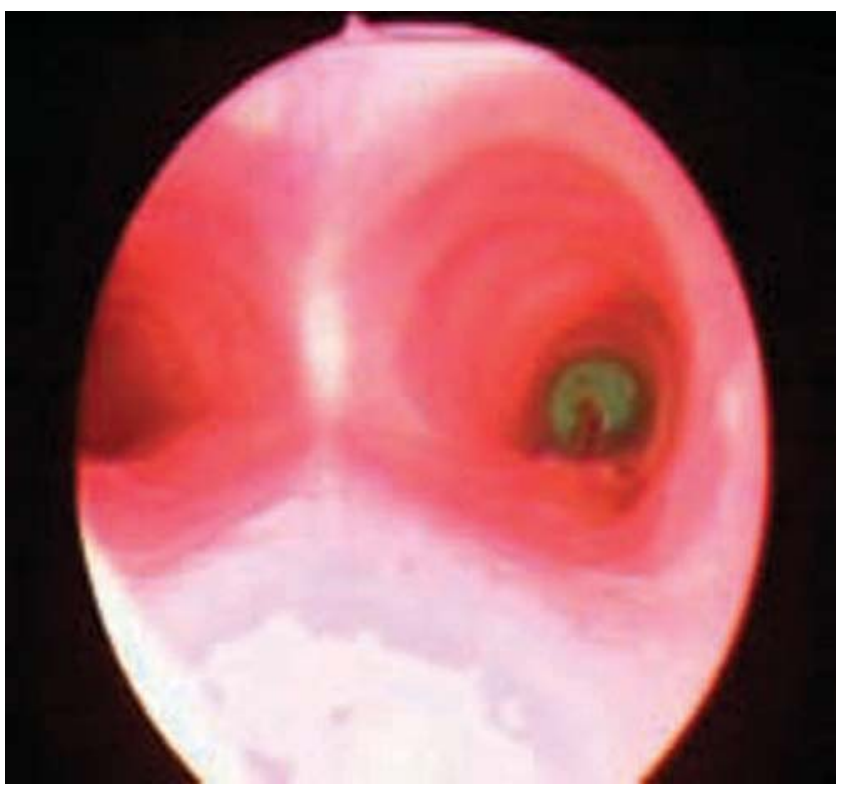

Figura 5. Observamos un alfiler con cabeza en bronquio derecho.

a nivel del cartílago cricoides, sobre todo en el lactante, cuya función epiglótica no está bien desarrollada. Cuando un cuerpo extrano se aloja en la glotis, de inmediato produce tos, atragantamiento y disfonía además de estridor y dificultad respiratoria. Si el cuerpo extraño se aloja en la abertura glótica, el niño presenta cianosis y lucha por respirar con mayor esfuerzo, momento en el cual está indicado realizar la maniobra de Heimlich ${ }^{2}$. Cuando el cuerpo extraño pasa a través de la glotis, se aloja en un bronquio o alguna de sus ramas y el cuadro clínico que ocasiona no es de insuficiencia respiratoria aguda ya que permite una ventilación parcial, la expresión clínica es tos y sibilancias, que puede tener varios días de evolución.

Cuando un cuerpo extraño lleva más de siete días alojado en la vía aérea, los síntomas que causa se atenúan y dificultan el diagnóstico y su extracción. El problema se confunde con un evento asmático agudo ${ }^{3}$. Sin embargo, se ha visto que los cuales mencionan que los síntomas más comunes en estos casos son la asfixia (76.8\%) y la tos prolongada $(14.3 \%)$ con una sensibilidad y especificidad del $91.1 \%$ y $42.5 \%$, respectivamente ${ }^{4}$. Hay que recalcar que el dato clínico que mejor orienta a sospechar que un paciente ha broncoaspirado un cuerpo extraño, es la información de un familiar, que a menudo relata que "el niño estaba jugando con algo en la boca y empezó a ahogarse". Esta información, cuando el niño es llevado al hospital, justifica realizar una broncoscopia diagnóstica, aún en ausencia de síntomas o alteraciones radiológicas.

Durante la exploración se debe observar la coloración de la piel (cianosis); cuantificar el grado de dificultad respiratoria: aleteo nasal, tiro intercostal, retracción subxifoidea, disociación toraco-abdominal y quejido espiratorio, asimetría a la inspección y realizar maniobras de amplexación y amplexión torácica que se verá disminuida en el hemitórax afectado; la percusión del tórax revelará hipersonoridad característica debida al atrapamiento de aire por un mecanismo de válvula que origina el cuerpo extraño y provoca sobredistension para compensar el lado afectado. Si se detecta matidez en lugar de hipersonoridad, se trata de atelectasia pulmonar. En la auscultación la disminución del murmullo vesicular del lado afectado es el dato más frecuente, pero pueden escucharse estertores, roncus o sibilancias.

Los síntomas pueden dividirse en tres fases. La primera fase corresponde al evento agudo, manifestado por crisis de asfixia, cianosis, dificultad respiratoria y tos de instalación súbita sin una patología respiratoria previa. En la segunda fase no existen síntomas y la exploración física no arroja anormalidades. Esta fase es la más peligrosa y la que más complicaciones tendrá posteriormente debido a que es fácil confundirla con un evento asmático y tratarla como tal, retrasando el tratamiento correcto.

En la tercera fase hay síntomas de infección respiratoria, con secreciones purulentas traqueobronquiales. Una radiografía de tórax puede mostrar imágenes sugerentes de atelectasias, neumonías, abscesos pulmonares o bronquiectasias ${ }^{5}$.

Los tipos de cuerpos extraños aspirados orgánicos (cacahuate, maíz, arroz, semillas de leguminosas, frijol, calabaza, huesos de pollo) ocasionan más complicaciones inflamatorias por su descomposición o fermentación. Son más difíciles de extraer por el aumento de volumen que sufren al contacto con la humedad de la vía aérea; se ocluyen por completo la luz de la vía respiratria. Los cuerpos extraños inorgánicos pueden variar desde alfileres, agujas, clavos, tornillos, tachuelas, tapón de lapiceros de plástico, restos de plástico de globos o pelotas, se localizan en el bronquio derecho (54.6\%), en el izquierdo (35\%), en la tráquea (10\%); en la subglotis (3\%); en ambos bronquios $(3 \%)^{6}$. Los que amenazan la vida en cuestión de minutos son los que se alojan en la tráquea, en ambos bronquios o en el espacio subglótico. 
Al sospechar la aspiración de un cuerpo extraño en vías aéreas se deben solicitar radiografías postero-anterior y lateral del tórax y tratar de visualizarlo en caso de que sea radiopaco.

Los datos que más orientan al diagnóstico son los debidos al atrapamiento de aire con hiperinsuflación debido al efecto de válvula por la elasticidad de los bronquios, que permite la entrada del aire pero no su salida. Cuando el atrapamiento de aire provoca un intenso esfuerzo de tos, puede causar ruptura alveolar que se manifestará con enfisema subcutáneo y en casos severos, con neumomediastino ${ }^{7}$.

La tomografía computarizada con reconstrucción bronquial ayuda a definir la localización exacta del cuerpo extraño en la vía aérea ${ }^{8}$. El procedimiento diagnóstico y terapéutico de elección más seguro es la broncoscopia rígida con anestesia general y tópica. Si el paciente tiene movimientos respiratorios adecuados se le permite permanecer con sus padres para evitar que el llanto pueda desplazar un cuerpo extraño impactado en un bronquio y que se desplace hacia la tráquea o a la subglotis y cause asfixia.

Hay que evitar hacer punciones venosas hasta llegar al quirófano y contar con el equipo anestésico, de intubación y reanimación cardiopulmonar avanzada. Los medicamentos para la anestesia general son midazolan, propofol y fentanil. Para anestesia tópica se usan la tetracaína o lidocaína. El trauma iatrogénico (aquel que se produce cuando el paciente no está relajado completamente y puede lesionarse la mucosa labial, la lengua o puede haber ruptura dental y llegar a la laceración bronquial) se puede reducir al relajar con vecuronio al paciente. Durante la broncoscopia es necesario un soporte ventilatorio para evitar la hipoxia, el laringoespasmo, la hipercapnia, la acidosis y el paro cardiaco. La administración de glucocorticoides previo al procedimiento reduce el riesgo de edema laríngeo 9. La broncoscopia rígida permite visualizar desde la epiglotis hasta la parte más distal de los bronquios; realizar la extracción con pequeñas pinzas; además logra ventilar al paciente al mismo tiempo ${ }^{10}$. El equipo consta de un laringoscopio de Jackson, broncoscopio rígido Storz tamaño acorde a la edad (Cuadro 1), fuente de luz, cable de fibra óptica, telescopio, aspirador, pinzas de cacahuate o canasta, ratón y caimán, además de pinza de canastilla para cuerpo extraño.
Cuadro 1.

\begin{tabular}{ccl}
\hline $\begin{array}{c}\text { Tamaño del } \\
\text { broncoscopio }\end{array}$ & $\begin{array}{c}\text { Diámetro } \\
\text { externo }\end{array}$ & \multicolumn{1}{c}{ Límites de edad } \\
\hline 2.5 & 4.0 & Prematuro y Neonato \\
3.0 & 5.0 & Neonato de 6 Meses \\
3.5 & 5.7 & 6-18 meses \\
4.0 & 7.0 & $18-36$ meses \\
5.0 & 7.8 & 3-8 años \\
6.0 & 8.2 & Mayores de 8 años \\
\hline
\end{tabular}

Técnica. La broncoscopia rígida se inicia colocando al paciente en decúbito dorsal, con la cabeza en ligera flexión; los hombros sobre la camilla; se protegen los dientes del paciente con los dedos del cirujano y se introduce el laringoscopio; se toma el broncoscopio como lápiz introduciéndolo bajo visión externa directa o bajo visión directa con telescopio, con el bisel dirigido lateralmente entre las cuerdas para separarlas; se le hace girar para abrirlas y se continua con la introducción rotando la cabeza del paciente en sentido opuesto al bronquio por examinar hasta localizar el cuerpo extraño. Una vez pinzado el cuerpo extraño, se retira la pinza hasta la punta del broncoscopio y se retiran ambos reunidos en concordancia con el anestesiólogo ${ }^{11}$. Después de retirar el cuerpo extraño, se debe revisar la vía aérea para descartar la presencia de otros cuerpos extraños o de restos del mismo; también para observar complicaciones como granulomas, úlceras y endobronquitis ${ }^{12}$.

En la actualidad existe un videobroncoscopio con un canal de trabajo interno con el que se realizan extracciones con mayor seguridad y firmeza, pero este equipo es sólo para adultos; tiene un canal interno en el cual puede introducirse la cámara y una entrada independiente para realizar la extracción bajo la visión de la cámara sin retirar el broncoscopio.

Los fibrobroncoscopios pediátricos sólo permiten el paso de canastillas o pinzas por el canal de trabajo de 1.1 $\mathrm{mm}$, lo que dificulta la extracción de objetos orgánicos por su densidad afectada por la humedad de la zona y pueden dañarse, romperse o fragmentarse ${ }^{13}$.

La vigilancia y el tratamiento postoperatorio deben incluir una radiografía de tórax, aerosolterapia con oximetazolina y broncodilatadores. Los antibióticos se emplean cuando el cuerpo extraño ha permanecido más de $72 \mathrm{~h}$ en el bronquio, cuando existe endobronquitis y hay fiebre.

Si no es posible extraer el cuerpo extraño mediante broncoscopia, raras veces se requiere hacer una traqueosto- 
mía ${ }^{14}$, toracotomía, broncotomía o lobectomía para extraer el objeto ${ }^{15}$. Las complicaciones más graves pueden ser neumotórax, neumonías con necrosis pulmonar, absceso pulmonar, bronquiectasias y fibrosis pulmonar ${ }^{12}$. La complicación más común en nuestros pacientes relacionada con el cuerpo extraño y no con el procedimiento, fue la endobronquitis (43\%); en otros estudios nacionales, fue el edema de glotis, no se menciona el porcentaje ${ }^{16}$.

Recomendaciones para evitar la aspiración de un cuerpo extraño: Evitar que los niños hablen o jueguen mientras coman; que se lleven objetos a la boca; darles juguetes de tamaño adecuado (mínimo $5 \mathrm{cc}$ ); evitar que duerman mientras chupan algo. Supervisarlos permanentemente.

\section{CONCLUSIONES}

Los cuerpos extraños en la vía aérea de los niños son una urgencia médica. La dificultad respiratoria, las crisis de asfixia, la polipnea y la cianosis son los síntomas principales. La broncoscopia rígida es un procedimiento diagnóstico y terapéutico seguro y eficaz en niños; las complicaciones atribuibles al procedimiento son prácticamente nulas, generalmente son causadas por el cuerpo extraño. En nuestro estudio no hubo complicaciones debidas al procedimiento por lo que corroboramos que la broncoscopia rígida es el procedimiento de elección en estos casos.

\section{BIBLIOGRAFÍA}

1. Siddiqui MA, Banjar AH, Al-Najjar M, et al. Frequency of tracheobronchial foreign bodies in children and adolescents. Saudi Med J. 2000;21:368-71.

2. Heimlich HJ, Patrick EA. The Heimlich maneuver. Best technique for saving any choking victim's life. Postgrad Med. 1990;87:38-53.
3. Schmidt $\mathrm{H}$, Manegold BC. Foreign body aspiration in children. Surg Endosc. 2000;14:644-8.

4. Lea E, Nawaf H, Yoav T, Elvin S, Zeév Z, Amir K. Diagnostic evaluation of foreign body aspiration in children: a prospective study. J Pediatr Surg. 2005;40:1122-7.

5. Quiroga OE, Ramil FC. Cuerpos extraños en vías aéreas. En: Casado J, Serrano A. Editores. Urgencias y tratamiento del niño grave. Madrid: Ergon; 2006. p. 341-4.

6. Soysal O, Kuzucu A, Ulutas H. Tracheobronchial foreign body aspiration: a continuing challenge. Otolaryngol Head Neck Surg. 2006;135(2):223-6.

7. Zerella JT, Dimler M, McGill LC, et al. Foreign body aspiration in children: value of radiography and complications of bronchoscopy: J Pediatr Surg. 1998;33:1651-4.

8. Huang HJ, Fang HY, Chen HC, Wu CY, Cheng CY, Chang $\mathrm{CL}$. Three-dimentional computed tomography for detection of tracheobronchial foreign body aspiration in children. Pediatr Surg Int. 2008;24:157-60.

9. Tomaske M, Gerber AC, Weiss M. Anesthesia and periinterventional morbidity of rigid bronchoscopy for tracheobronchial foreign body diagnosis and removal. Paediatr Anaesth. 2006;16(2):123-9.

10. Hui H, Na L, Zhijun CJ, Fugao ZG, Yan S, Niankai ZK, Jingjing CJ. Therapeutic experience from 1428 patients with pediatric tracheobronchial foreign body. J Pediatr Surg. 2008:43;71821.

11. Gans SL. Bronchoscopy. En: Gans SL (Ed). Pediatric Endoscopy. Los Angeles: 1983. p. 37-54.

12. Mu L, He P, Sun D. The causes and complications of late diagnosis of foreign body aspiration in children. Report of 210 cases. Arch Otolaryngol Head Neck Surg. 1991;117:876-9.

13. Swanson KL, Prakash UB, Midthun DE, et al. Flexible bronchoscopic management of airway foreign bodies in children. Chest. 2002;121:1965-700.

14. Baharloo F, Veychkemans F, Biettlot MP, et al. Tracheobronchial foreign bodies: Presentation and management in children and adults. Chest. 1999;115:1537-61.

15. Ülku R, Onen A, Onat S, Ozcelik C. The value of open surgical approaches for aspirated pen caps. J Pediatr Surg. 2005;40:1780-83.

16. Gómez-Leyva E, Chávez-Enriquez EP, Domínguez-Cadena A, Alvarado-León U, Victoria-Zuñiga JD. Cuerpos extraños intratorácios. Presentación de cinco casos. Arch Inv Mat Inf. 2009;1:(1):14-7. 